\title{
Assimilationniste « de couleur » contre autonomisme «blanc »
}

De la Révolution à l'abolition de l'esclavage de 1848

David Rigoulet-Roze

\section{(2) OpenEdition \\ Journals}

Édition électronique

URL : http://journals.openedition.org/plc/725

DOI : $10.4000 /$ plc. 725

ISSN : 2117-5209

Éditeur

L'Harmattan

Édition imprimée

Date de publication : 1 janvier 1997

Pagination : 78-109

ISSN : 1279-8657

Référence électronique

David Rigoulet-Roze, «Assimilationniste « de couleur » contre autonomisme « blanc » », Pouvoirs dans la Caraïbe [En ligne], Spécial | 1997, mis en ligne le 15 mars 2011, consulté le 20 avril 2019. URL: http://journals.openedition.org/plc/725; DOI : 10.4000/plc.725 


\section{Assimilationniste « de couleur » contre autonomisme « blanc » : de la Révolution à l'abolition de l'esclavage de 1848}

par David RIGOULET-ROZE

Doctorant en géopolitique

Université Paris VIII

\section{INTRODUCTION}

Alors que l'on célèbre, en 1998, le cent-cinquantenaire de l'abolition de l'esclavage, cette commémoration offre l'occasion de revenir sur le long processus assimilationniste à la française qui l'a précédé. L'idée d'assimilation, qui va trouver plus tard son achèvement juridique dans la départementalisation de 1946, n'était pas une idée neuve à l'époque, et elle n'a pas toujours été d'ailleurs l'apanage des seuls courants progressistes. On ne sait pas toujours à quel point cette idée a pu, dans l'Histoire, se trouver opportunément instrumentalisée par le pouvoir métropolitain, pour éviter que le problème de l'autonomie ne soit effectivement posé. Il s'agit donc d'un problème complexe qu'il convient de traiter avec beaucoup de nuances pour déterminer comment et pour quelles raisons les courants progressistes ont été amenés, à partir de la Révolution française, à investir cette logique assimilationniste. 


\section{L'ASSUJETTISSEMENT DES ANTILLES \\ PAR L'ETAT MONARCHIQUE SOUS L'ANCIEN REGIME}

Certains ont pu dire que la France en avait fait sa politique dès avant la Révolution française. Mais si c'est le cas, ce fut avec des attendus bien différents selon les uns et les autres, sinon opposés.

Sous l'Ancien Régime, les colonies étaient juridiquement perçues comme une extension du territoire national, comme des « provinces de la mère patrie », et à première vue, l'administration de ces territoires par le pouvoir royal relevait d'une logique assimilationniste. En réalité, les choses étaient plus complexes car, en fait d'assimilation, c'est plutôt l'esprit de tutelle, caractérisé par le souci du contrôle tatillon et la subordination aux intérêts économiques de la métropole - via le régime protectionniste de l'Exclusif -, qui semble avoir constitué la dominante de toute la politique monarchique. Les colonies n'étaient pas des territoires comme les autres.

Si les institutions coloniales reproduisaient plus ou moins celles de la métropole, sous l'Ancien Régime cela n'impliquait cependant aucune espèce d'égalité entre la France et ses territoires d'Outre-Mer. Une colonie ne se différenciait peut-être pas du reste du royaume par un statut administratif particulier mais, en tout cas, par un régime économique très contraignant garantissant à la métropole un système d'exploitation à son unique profit. C'est dire que la fonction première de la monarchie absolue, en tant qu'Etat colonial, consistait essentiellement à maintenir les Antilles dans le domaine mercantile français. Et c'est en fait à la Révolution française que l'assimilation à été plus qu'un simple cadre administratif.

Il ne faut donc pas surévaluer les tendances à l'assimilation manifestées avant la Révolution par le pouvoir métropolitain à l'égard des colons, et ne pas perdre de vue qu'il est tout simplement inconcevable que le principe de l'assimilation, en tant que tel, ait pu être au cœur de la politique coloniale française, ne serait-ce que pour la raison suivante : la ségrégation raciale et la discrimination juridique aux Antilles apparaissent comme co-extensives de la colonisation. 
Dans les faits, les Blancs installés aux Antilles avaient toujours bénéficié des mêmes droits, privilèges et immunités que les Français de la métropole. De ce point de vue, leur assimilation à la métropole n'était pas sujette à caution, elle allait de soi. Tel n'était évidemment pas le cas, en revanche, pour les «hommes de couleur libres», et moins encore pour les esclaves noirs à qui la citoyenneté ne sera finalement accordée en droit qu'en 1848. Dans cette perspective, la Révolution française constitua bien une rupture fondamentale. Sans la République, la citoyenneté politique n'aurait jamais été étendue ni aux « hommes de couleur libres » ni aux esclaves. Elle aurait été réservée aux seuls Blancs comme cela avait toujours été le cas jusque-là.

\section{LES HESITATIONS DE LA POLITIQUE COLONIALE DURANT LES PREMIERES ANNEES DU REGIME REPUBLICAIN ISSU DE 1789}

Contrairement aux idées répandues, la Révolution française, ne fut pas toujours résolument «assimilationniste », jusque et y compris dans sa phase jacobine - laquelle fut d'une durée somme toute assez réduite (juin 1793 - juillet 1794).

Dès l'abolition des privilèges, proclamée la nuit du 4 août 1789 , qui fut prolongée par la «Déclaration des droits de l'homme et du citoyen » votée le 26 août, les «libres de couleur» ainsi que les esclaves pensèrent, en toute logique, que le roi leur avait accordé la liberté et l'exercice des droits afférents au même titre que tous les autres sujets du Royaume de France; si cette égalité n'était pas rendue effective dans les colonies, la faute ne pouvait en incomber qu'aux colons qui la leur refusaient. En réalité, les choses étaient plus complexes. Non pas en ce qui concerne l'attitude des dits colons dont on avait rien d'autre à attendre, mais de la part de l'Assemblée constituante qui recherchait pour les îles un bien improbable statut ne remettant pas forcément en cause l'empire colonial français.

Etrangement, ceux qui furent les hérauts du processus révolutionnaire furent, au moins au début de la période révolutionnaire, largement favorables à l'autonomie des colonies. Ils soutenaient, dans 
une logique différentialiste, l'idée que les colonies devaient s'appartenir et se gouverner par elles-mêmes. Un paradoxe qui n'en est pas forcément un.

En effet, l'Assemblée Constituante de 1790 estima qu'il serait inadéquat de donner à l'Assemblée coloniale auto-constituée en octobre 1789, et immédiatement chargée d'élaborer un projet constitutionnel, des instructions détaillées en la matière ; et surtout, que cela altérerait «l'esprit du décret rendu en faveur des colonies en faisant fi, pour ainsi dire d'avance, de la constitution qu'elles (étaient) invitées à proposer ». La dynamique de la Révolution française accentuait, malgré elle, si l'on peut dire, dans un premier temps l'autonomisme colon. Le raisonnement des colons, quant à eux, était le suivant : à partir du moment où la métropole était appelée à se doter d'un régime politique à sa convenance, au nom de quoi empêcherait-on les colonies de faire de même ? Ils revendiquèrent donc une autonomie législative en association avec la France. Et c'est ce qu'ils obtinrent de la Constituante.

Par le décret du 8 mars 1790, la Constituante octroya l'autonomie aux possessions d'Outre-Mer, et en remit l'exercice à leurs «citoyens actifs», c'est-à-dire propriétaires et payant une contribution. Rien n'était précisé concernant les « hommes de couleur libres ». Or, nombre d'entre eux répondaient aux conditions requises pour être «citoyens actifs » et par conséquent pour voter ou être éligibles. Au sein de la Constituante, certains par la voix de Mirabeau, avaient tenté de protester contre une terminologie floue dans laquelle chacun pouvait lire ce qui lui convenait. De la sorte, les planteurs se sentirent en droit d'affirmer qu'ils étaient les seuls «citoyens» des colonies; tandis que les «gens de couleur libres » assuraient à juste titre qu'ils appartenaient désormais à cette nouvelle classe puisqu'ils jouissaient de la liberté. Aussi, dès le 10 mars, ces derniers déposèrentils des Réclamations sur le bureau du parlement national. Une réponse définitive leur fut apportée le 28 mars, dans des Instructions complémentaires au décret du 8 mars, concoctées par Barnave, un ami dévoué des Blancs et proche du Club Massiac représentant le lobby colon. Le corps électoral des colonies fut défini comme «l'ensemble des personnes âgées de 25 ans accomplis, propriétaires d'immeubles, 
ou à défaut, d'une telle propriété, domiciliées dans la paroisse depuis deux ans et payant une contribution ». Cette disposition qui prolongeait la confusion du décret du 8 mars, provoqua un débat houleux. L'Abbé Grégoire critiqua son imprécision et réclama que l'on mentionnât au moins expressément la présence des «Gens de couleur libres » dans le collège des électeurs et des éligibles. Barnave intervint alors et certifia que les termes de l'Instruction ne les excluaient pas. L'Abbé Grégoire, apparemment satisfait par cette mise au point, n'insista pas. Les représentants des planteurs, pour leur part, protestèrent que, de toute façon, jamais les colons n'accepteraient de reconnaître le droit de représentation aux « hommes de couleur » fussent-ils libres.

Les termes de ce débat étaient largement biaisés et réduisaient en fait à néant toutes les espérances induites par 1789. Les règles normatives formulées en ce mois de mars 1790 découlaient entièrement de l'idéologie des colons. Elles établissaient clairement l'autonomie coloniale, une vieille revendication de la classe dirigeante coloniale, mais n'empêchaient pas, en réalité, d'écarter les «Gens de couleur libres ». Les colons avaient finalement obtenu satisfaction : s'ils étaient prêts à faire un certain compromis avec Paris, notamment sur le régime extérieur des colonies, ils n'entendaient en aucune manière transiger sur le terrain de l'égalité des « hommes de couleur libres », pour ne pas parler du problème encore plus tabou de l'esclavage.

S'efforçant de tirer parti de l'ambiguïté inhérente aux décisions de l'Assemblée constituante, les «Gens de couleur libres » étaient, quant à eux, bien décidés à faire valoir la lettre de la loi en ignorant délibérément l'esprit ambigu qui sous-tendait son cheminement doctrinal, et affirmer que, quoique puissent en dire les colons, la législation des 8 et 28 mars leur avait bien reconnu l'égalité politique avec les Blancs. Ils revendiquaient ainsi une assimilation pleine et entière au monde européen, avec toutes les conséquences que cela impliquait.

L'assimilation allait, dès lors, se présenter comme la réponse des « gens de couleur » à l'autonomisme ségrégationniste et oppressif des colons. Ils ne faisaient là que pousser dans ses ultimes conséquences l'idéologie universaliste de 1789, même si la Révolution, curieusement 
conservatrice par certains aspects, tarda à concrétiser les espoirs qu'elle avait pu susciter.

Ces hésitations doctrinales entre les attendus de la «Déclaration des droits » d'août 1789 et le caractère différé de son application dans les îles constitua une constante durant les premières années de la période révolutionnaire. Or, l'Assemblée constituante était déjà sous la pression d'événements qui se produisirent aussi bien en métropole que dans les colonies - notamment à Saint-Domingue avec l'insurrection, fin octobre 1790, des «hommes de couleur» et affranchis à SaintDomingue, précisément devant le refus des Blancs de leur appliquer le décret du 8 mars. Cette tentative de soulèvement échoua et la répression s'abattit sur les chefs du mouvement insurrectionnel, Vincent Ogé et J.-B. Chavannes, mais l'agitation subsista durablement dans tout le pays. Ce n'était que partie remise pour l'année suivante.

A la tribune de l'Assemblée constituante, appuyés par une partie de l'opinion publique, les «Amis des Noirs» ne cessaient pourtant de poser le problème des «hommes de couleur». Bon nombre de séances furent consacrées aux colonies. Pour l'heure, il ne pouvait encore être question de l'abolition de l'esclavage. L'Assemblée constituante se préoccupait surtout de la situation des «hommes de couleur libres ». Il lui était évidemment difficile de soutenir que des gens, propriétaires, instruits, et participant aux guerres de la Métropole, et qui plus est, payant des impôts, n'étaient pas des hommes. Dans ces conditions, la «Déclaration des droits de l'homme et du citoyen » ne pouvait pas ne pas les concerner, ce qui impliquait qu'on leur donnât l'égalité civique et politique. De la théorie à la réalité, il y avait cependant un fossé à combler. L'Assemblée pressentait que toucher au système colonial, même a minima, équivaudrait à bouleverser la société esclavagiste et à ouvrir, à terme, la voie à la liberté des esclaves. Or, l'esclavage constituait encore l'un des piliers essentiels de la richesse des colonies dont bénéficiait la métropole. Le remettre en cause, c'était risquer de perdre les colonies. L'Assemblée constituante n'arriva jamais à surmonter ces contradictions et ses différentes volte-face ne firent qu'accroître les tensions. 
En mai 1791, le débat revint devant l'Assemblée constituante. Ce fut sans conteste le plus important de la législature et celui qui illustre jusqu'à la caricature les contradictions qui traversaient cette Assemblée. Alors que sur la proposition de Barnave, la Constituante venait tout juste de décréter qu' «aucune loi sur l'état des personnes non libres ne pourra être faite pour les colonies, que sur la demande formelle et spontanée des assemblées coloniales », un amendement l'amendement Reubell - fut inopinément adopté, donnant les droits politiques aux mulâtres nés de père et de mère libres. Grâce à la lutte vigoureuse de l'Abbé Grégoire, de Brissot et de Condorcet, ce qui devint le décret du 15 mai 1791 reconnaissait officiellement l'égalité politique, même si c'était de façon restrictive puisqu'elle ne concernait que les Hommes de couleur nés de parents libres, et non ceux devenus libres après affranchissement ou ceux dont l'un des parents n'étaient pas libres. Ce décret ne concernait donc que quelques centaines de personnes de couleur libres alors que ceux-ci étaient déjà entre $20 \%$ et $40 \%$ de la population aux colonies et aussi nombreux que les Blancs à Saint-Domingue. Néanmoins, la brèche était ouverte dans la muraille coloniale.

Les détracteurs du projet ne s'y trompèrent d'ailleurs pas. Ce vote provoqua la fureur des députés colons devant ce qu'ils percevaient comme une bombe à retardement. Ils brandirent l'épouvantail de la perte des colonies, de l'insurrection des esclaves. Ils se virent confortés dans cette idée par le soulèvement des esclaves à Saint-Domingue le 22 août 1791, après lequel l'ordre colonial n'allait plus pouvoir être rétabli.

Cet événement explique sans doute les ultimes mesures « conservatrices » prises par l'Assemblée constituante. Deux pas en avant, un pas en arrière semble, en effet, avoir constitué la dominante de la politique métropolitaine à l'égard des colonies. Dans la rédaction de la Constitution du 3 septembre 1791 largement inspirée par Barnave. Il a beau être énoncé, en préalable, que l'Assemblée Nationale voulant établir la Constitution sur les principes qu'elle vient de reconnaître et de déclarer, abolit irrévocablement les institutions qui blessaient la liberté et l'égalité des droits (et qu'il) «n'y a plus, pour aucune partie de la Nation, ni pour aucun individu, aucun privilège, ni 
exception au droit commun de tous les Français », le seul article consacré aux colonies dispose pourtant que «Les colonies et possessions françaises dans l'Asie, l'Afrique et l'Amérique, quoiqu'elles fassent partie de l'Empire français, ne sont pas comprises dans la présente Constitution » (Titre III, chap. 3, sect. I, art. 1). La contradiction était patente. Mieux encore, le 24 septembre, alors que la Constitution se trouvait déjà promulguée, l'Assemblée, sous la pression du lobby de la plantocratie créole et de ses appuis au sein de la Constituante, vota un nouveau décret qui revenait sur les dispositions du 15 mai et donnait toute latitude aux Assemblées coloniales, exclusivement composées de Blancs, pour les questions concernant «l'état des personnes non libres et l'état politique des Hommes de couleur et des Nègres libres ». Tout dépendait désormais du bon vouloir des colons blancs qui avaient tout fait pour interdire l'entrée des «Hommes de couleur libres » dans les assemblées coloniales.

\section{LA CONSECRATION PAR L'ASSEMBLEE LEGISLATIVE DE L'EGALITE DES DROITS POUR LES « GENS DE COULEUR LIBRES » DANS LES COLONIES}

C'est en réalité à l'Assemblée législative - qui siégea entre octobre 1791 et août 1792, et où l'on retrouve beaucoup d'Amis des Noirs et en particulier Brissot qui en fut, un certain temps, le leader de la majorité - qu'il revint de tirer les leçons des erreurs et hésitations de la Constituante au sujet des « hommes de couleur libres ». Le problème colonial fut reconsidéré avec plus de réalisme. Pour conserver ses colonies, la France devait justement passer outre aux résistances suicidaires des colons et accorder l'égalité des droits politiques à tous les hommes de couleur libres. Le raisonnement était désormais fait sien par une majorité de députés, y compris parmi ceux qui étaient peu suspects de sympathie pour les «Amis des Noirs ». L'insurrection de Saint-Domingue ne fut pas étrangère à cette prise de conscience. A Paris, on commençait à comprendre que l'adoption des mesures les plus « conservatrices » sinon « réactionnaires » ne faisait qu'accroître l'agitation dans les colonies, avec le risque de les perdre définitivement. 
Cette insurrection des esclaves pesa de plus en plus lourdement durant toute l'année 1792 sur la politique de la France vis-à-vis de ses colonies. Cette insurrection risquait de s'étendre aux autres colonies. Cela explique pour une bonne part les mesures prises par l'Assemblée législative en faveur des «libres de couleur» au début de l'année 1792. Il reste que l'Assemblée fut à chaque fois en retard par rapport aux faits. Le problème n'était déjà plus celui des libres de couleur mais déjà celui de la reconnaissance de la liberté des esclaves.

Sur le rapport de Gensonné, l'Assemblée législative adopta le décret du 28 mars 1792, promulgué le 4 avril de la même année, lequel accordait aux «Gens de couleur libres » les mêmes droits électoraux qu'aux Blancs. Le 3 juin 1792, pour éviter de nouveaux problèmes, l'Assemblée coloniale de la Martinique accepta le décret de la Législative même si elle ne se hâta pas de l'appliquer. Les esclaves noirs étaient encore ignorés, mais ils s'étaient déjà mis en mouvement dans les îles au nom de cette «liberté » proclamée le 26 août 1789.

Force est de constater, que plus de trois ans de maturation des esprits avaient été nécessaires avant que l'Assemblée législative ne consacrât, juridiquement parlant, l'égalité inscrite dans la « Déclaration des Droits » de 1789.

\section{LA « PREMIERE » ABOLITION DE L'ESCLAVAGE PAR LA CONVENTION MONTAGNARDE}

Deux ans supplémentaires furent encore nécessaires avant de mettre en pratique le second des grands principes universalistes de cette «Déclaration», la liberté. Il faut dire qu'entre temps, il s'était produit un certain nombre d'événements : la chute de la Monarchie en août 1792 suivie de la proclamation de la République, et la Législative remplacée par une nouvelle assemblée, la Convention jacobine, plus révolutionnaire que la précédente C'est en effet la Convention qui décréta l'abolition de l'esclavage, le 16 Pluviôse an II (4 février 1794), confirmant les décisions des commissaires de la République prises de leur propre initiative à Saint-Domingue à la fin de l'été 1793. A la réserve près que ladite Convention, par la voix de Danton, avait ajouté 
prudemment que l'on renvoyait au Comité colonial le soin d'arrêter les mesures d'application de la loi Pluviôse an II abolissant l'esclavage. «La Convention vient de faire son devoir. Mais après avoir accordé le bienfait de la liberté, il faut que nous en soyons pour ainsi dire les modérateurs. Renvoyons aux comités de salut public et des colonies, pour combiner les moyens de rendre ce décret utile à l'humanité sans aucun danger pour elle $\aleph^{1}$. Autre façon de dire que cette reconnaissance n'allait pas forcément de soi pour tous les conventionnels.

Comment alors interpréter le vote de la Convention Montagnarde, elle qui avait confirmé l'égalité aux «hommes de couleur libres », mais qui, à l'exception de quelques-uns de ses membres comme le Père Duschesne, l'Abbé Grégoire, était restée jusque-là plutôt hostile à l'abolition de l'esclavage ? Pour le comprendre, il n'est certainement pas inutile ici de revenir plus avant sur l'arrière-plan géopolitique qui avait finalement conduit, bon gré mal gré, à l'adoption de ces mesures : en l'occurrence, sur la situation chaotique qui prévalait à Saint-Domingue, fleuron des colonies françaises aux Antilles. Dans le prolongement du premier soulèvement des « gens de couleur libres » que l'île avait connu dès 1790, s'était déclenchée la grande insurrection des esclaves dans la nuit du 22 au 23 août 1791. Cette insurrection n'avait cessé de prendre de l'ampleur durant l'année 1792, pour devenir un problème majeur à partir de l'année 1793.

Sur le moment, les colons blancs, qui sous-estimaient les révoltés noirs, accusèrent les « gens de couleur » d'avoir fomenté cette révolte. En réalité, cette insurrection s'inscrivait directement dans le prolongement $\mathrm{du}$ processus révolutionnaire à l'œuvre à Paris. La situation locale avait quelque chose de surréaliste. Les Blancs, en effet, rejetaient par principe la loi du 4 avril 1792 et l'égalité politique qu'elle instaurait, tandis que les «gens de couleur libres » l'invoquaient à grands cris - et cela, sans que fût jamais posé pour autant le problème de l'esclavage ! Or, celui-ci devenait, pratiquement parlant, de plus en plus ineffectif puisque les Noirs avaient cessé

1. Cf. Aimé Césaire, Toussaint Louverture, Paris, Présence Africaine, 1981, p. 219. 
presque partout de travailler et combattaient les armes à la main pour leur liberté.

Ce fut dans ce contexte devenu explosif pour les îles que la Convention déclara la guerre à l'Angleterre le $1^{\text {er }}$ février 1793 et à l'Espagne le 7 mars de la même année. Pressées par les colons qui leur opposaient des troupes dirigées par l'ex-Gouverneur Galbaud et par la menace des interventions militaires espagnole et anglaise, les deux commissaires de la République, Sonthonax, partisan déclaré de l'abolition, et son homologue Polverel, craignant « de voir passer dans les mains ennemies la propriété de Saint-Domingue », se décidèrent à décréter, respectivement le 29 août 1793 dans la province du nord et le 4 septembre dans les parties ouest et sud, une première émancipation des esclaves. Avec l'espoir que cette mesure fournirait des recrues aux troupes françaises engagées dans la défense du territoire colonial. Les autorités coloniales pensaient qu'une telle proclamation convaincrait les Noirs des bonnes intentions du gouvernement et partant, du changement irréversible de leur condition.

Cette transformation de leur condition, ils la devaient en fait à leur rébellion qui durait depuis deux ans déjà. Il faut rappeler ici le rôle déterminant et largement sous-évalué dans l'histoire coloniale française de Toussaint Louverture. Comme le rapporte Aimé Césaire dans son Toussaint Louverture, ce dernier apparaît à Saint-Domingue «pour prendre à la lettre la déclaration des droits de l'homme, (...) pour montrer qu'il n'y a pas de race paria ; qu'il n'y a pas de pays marginal; qu'il n'y a pas de peuple d'exception $»^{2}$. De fait, c'est lui qui sut exiger des commissaires de la République, avant le ralliement définitif de ses troupes aux forces républicaines qui interviendra en mai 1794, que cette abolition décrétée dans l'urgence en 1793 fût ratifiée par la Convention montagnarde, laquelle (avec seulement 180 députés présents) ne fit, somme toute, que confirmer et généraliser cette abolition par la loi du 16 Pluviôse An II (4 février 1794) :

«La Convention nationale déclare abolir l'esclavage des Nègres dans toutes les colonies. En conséquence, elle décrète que les hommes, sans distinction de couleur, domiciliés dans les colonies, sont citoyens

${ }^{2}$. Cf. Aimé Césaire, Toussaint Louverture, Paris, Présence Africaine, 1981, p. 344. 
français et jouiront de tous les droits assurés par la Constitution. Renvoie au Comité de salut public pour lui faire incessamment un rapport sur les mesures à prendre de l'exécution du décret ».

Il y eut de la part des autorités coloniales, une grande part d'opportunisme politique dans cette décision, car il s'agissait surtout de faire pièce à un autonomisme colon devenu franchement séparatiste puisqu'il était prêt à livrer les îles aux ennemis de la République pour maintenir ses privilèges.

Cependant, seules trois colonies purent bénéficier de cette mesure : Saint-Domingue évidemment, la Guadeloupe reconquise par le républicain Victor Hugues qui proclama l'abolition le 7 juin 1794, et la Guyane française quelque peu épargnée par la tourmente de la guerre. En effet, les Anglais s'emparèrent de la Martinique en mars 1794 - précisément avec l'appui des colons autonomistes -, et ne la rendirent une première fois aux Français, avec Sainte-Lucie et Tobago, qu'à l'issue de la Paix d'Amiens en 1802.

\section{LA CONSTITUTION THERMIDORIENNE ET LA « PREMIERE DEPARTEMENTALISATION »}

Il fallut attendre l'aggravation des difficultés intérieures et extérieures de la Révolution pour voir s'esquisser une nouvelle politique coloniale en rupture avec les hésitations, sinon les orientations autonomistes, des premières années du régime républicain. Là encore, aussi étonnant que cela puisse paraître, ce n'est pas le jacobinisme centralisateur des Montagnards, lesquels viennent tout juste d'être guillotinés - la Constitution de Robespierre ayant été suspendue dès sa proclamation, la France n'a jamais connu le jacobinisme dont elle se réclame - mais les Thermidoriens qui introduisirent la dimension assimilationniste dans la politique coloniale française. Ils firent voter la première loi constitutionnelle du 5 fructidor an III (22 août 1795) disposant que les «colonies françaises sont parties intégrantes de la République et sont soumises à la même loi constitutionnelle » et tendant à transformer les vieilles colonies en départements. La nouvelle constitution ne fut jamais appliquée à la 
Martinique qui était passée aux Anglais en mars 1794. Cependant il convient de rappeler qu'il y avait déjà eu, dans l'île, un précédent de cette nature puisque le 26 octobre 1793, l'assemblée représentative de la Martinique, élue sous le Gouverneur républicain Rochambeau, avait voté la transformation de la colonie en « département ». L'île avait été livrée aux Anglais par les Blancs royalistes qui étaient autonomistes, alors que les Républicains voulaient déjà l'assimilation avec la métropole parce qu'elle induisait les mêmes droits, les mêmes devoirs et les mêmes institutions.

La Constitution de l'An III apparaît bien comme le point de départ de la politique d'assimilation à la française et le rapport préparatoire de Boissy d'Anglas devant la commission thermidorienne comme le document fondateur de la doctrine assimilationniste. C'est cette volonté «d'assimiler en tout les colonies aux autres parties de la République » (François Antoine Boissy d'Anglas, 1795) qui devint plus tard la politique traditionnelle de la France à l'égard des Antilles.

Il n'est pas inutile de revenir sur les termes du débat. On discuta des colonies au cours de longues discussions qui précédèrent le vote de la Constitution de l'an III (22 août 1795), la seule qui se soit étendue aux dites colonies. Boissy d'Anglas, le rapporteur de la «Commission des Onze », fut chargé de préparer le projet constitutionnel. Dans ses «rapport et projet d'articles constitutionnels relatifs aux colonies présentés à la Convention Nationale au nom de la Commission des Onze » le 17 Thermidor an III (4 août 1795), Boissy d'Anglas exposa les thèses de l'assimilation et de la domination de la France.

« Rattachons les colonies à nous, par un gouvernement sage et ferme, par les liens d'un intérêt commun, par l'attrait puissant de la liberté. Que les colonies soient toujours françaises, au lieu d'être seulement américaines ; qu'elles soient libres, sans être indépendantes ; qu'elles fassent partie de notre république indivisible, et qu'elles soient surveillées et régies par les mêmes lois et le même gouvernement; que leurs députés, appelés en cette enceinte, y soient confondus avec ceux du peuple entier, qu'ils seront chargés de représenter ». 
A cette occasion, prenait naissance un courant jacobin concernant la souveraineté nationale et la mainmise sur les possessions françaises :

De fait, l'article VI de la Constitution de l'An III disposait que :

«Les colonies françaises font partie de la République et sont soumises à la même loi constitutionnelle ».

Et l'article VII décrétait :

«Elles sont divisées en départements ».

C'est si l'on peut dire, la «première départementalisation »des Antilles. L'assimilation était conçue par les Conventionnels comme un moyen de combattre les orientations centrifuges des assemblées coloniales. Pelet, membre du Comité de Salut Public, avait déclaré ouvertement le 23 janvier 1795 : «Les colonies seront sujettes, soumises et non indépendantes de la République ; elles resteront (...) invariablement et indivisiblement réunies à la France ».

La Convention thermidorienne s'efforçait, en fait, de faire triompher la tendance unitaire de la République en préconisant l'assimilation totale qui passait par l'application du régime administratif et de la législation métropolitaine aux colonies dotées dorénavant d'une représentation aux assemblées législatives centrales.

Mais les troubles persistants rendirent inapplicable ces décisions. La nouvelle architecture institutionnelle ne résista pas aux difficultés exceptionnelles de cette période. D'ailleurs, en pratique, de nombreuses lois spéciales accentuèrent progressivement les ingérences du Directoire dans les affaires courantes. Dans l'échec de la mise en œuvre de la Constitution de l'an III, se dessina la « réaction » à la fois à travers la centralisation napoléonienne en métropole, et le retour à un régime d'exception pour les possessions françaises d'Amérique. 


\section{LE RETOUR AU REGIME D'EXCEPTION SOUS L'EMPIRE ET LE RETABLISSEMENT DE L'ESCLAVAGE}

Le coup d'Etat du 18 Brumaire an VIII (8 novembre 1799) mit fin au Directoire, et avec le Consulat et l'Empire, ce fut le retour à l'assujettissement pour les îles antillaises. La constitution du 22 Frimaire an VIII (13 décembre 1799) disposa dans son article 91 :

«Le régime des colonies est déterminé par des lois spéciales ».

Ce n'était rien moins qu'une rupture avec la politique d'assimilation, mais ce n'était pas pour autant une évolution statutaire vers une autonomie. Les colonies furent désormais soumises au régime des décrets; la représentation coloniale disparut et on rétablit les anciennes chambres d'agriculture dont les membres étaient nommés et non élus; avec des noms différents, un Capitaine général héritait des pouvoirs de l'ancien gouverneur et un préfet colonial de ceux de l'intendant. Il ne fut ainsi plus question de représentation coloniale pendant un demi-siècle. Et, pour parachever le tout, Bonaparte décida de rétablir l'esclavage et la traite dans les îles. Autant dire que le débat sur l'assimilation fut relégué aux oubliettes.

Si la récupération de la Martinique, en juillet 1802, ne posa pas de problèmes insolubles, sans doute parce que, devenue un temps anglaise, elle n'avait pas connu l'application du décret abolitionniste de 1794, les choses furent moins aisées à la Guadeloupe. Le rétablissement de l'ordre esclavagiste provoqua une insurrection des esclaves dirigée par Delgrès, qui échoua. Mais le souvenir perdura que la France avait pu concevoir les colonies sans l'esclavage.

Mais surtout l'esclavage ne put être rétabli dans toutes les possessions françaises puisque la métropole ne devait jamais récupérer Saint-Domingue où les esclaves émancipés combattirent les armes à la main pour défendre une liberté qu'ils avaient gagnée au prix fort depuis près de dix ans.

A Saint-Domingue, en effet, l'émancipation des esclaves en 1793, le ralliement à la France républicaine de Toussaint-Louverture, 
un des principaux chefs de l'insurrection, en 1794, avaient créé dans la perle des colonies françaises une situation radicalement nouvelle pour la métropole. Très rapidement, Toussaint-Louverture avait imposé son autorité. Il avait même dans un premier temps chassé les Espagnols en 1795 de la moitié est de l'île - une situation entérinée par le traité de Bâle (24 juillet 1795). Et, promu par le Directoire, Général en chef de l'armée de Saint-Domingue en avril 1796, il était finalement parvenu à repousser les Anglais en mai 1798. Il se permit le luxe de signer avec Londres un traité de commerce et de neutralité, qui ouvrait les ports de la colonie au commerce nord-américain. Il enjoignit les agents de la puissance coloniale qui contrecarraient ses plans à réembarquer pour la France. A l'issue d'une guerre civile qui dura un an (1799-1800), il brisa la puissance rivale des anciens libres de couleur qui avaient à leur tête le général André Rigaud. Il fit élire une Assemblée constituante qui promulgua une Constitution le 8 juillet 1801 et fut enfin proclamé gouverneur général de Saint-Domingue au nom de la France.

Comme le relève Aimé Césaire dans son Toussaint-Louverture : on a parlé de l'ambition de Toussaint. Ce n'est pas de psychologie qu'il s'agit ici. C'est de tout autre chose, de la force des événements et de la poussée historique. Et c'est sur cette poussée que Toussaint avait l'œil rivé. Et c'était elle, avec ses tempêtes, ses tourbillons, ses raz-demarée, ses coups de boutoir suivis de calme, qui inquiétaient Toussaint. La France allait-elle revenir sur le décret d'abolition de l'esclavage ? Là était la crainte de Toussaint ${ }^{3} \gg$. Cette crainte n'était pas infondée car Bonaparte ne pouvait supporter la place que Toussaint-Louverture avait prise dans la Grande île.

Bonaparte voulant rétablir l'esclavage à Saint-Domingue comme dans les autres îles françaises, il avait chargé après la Paix d'Amiens de 1802, une force expéditionnaire importante d'y restaurer les anciennes structures coloniales. Conscient que le rapport de forces sur le terrain n'allait pas de soi, il avait donné aux différents chefs de l'expédition des instructions, qu'ils devaient garder secrètes pendant un certain temps pour tromper les populations : les instructions secrètes de Leclerc, chargé de reprendre Saint-Domingue - à l'instar de celles

3. Cf. Aimé Césaire, Toussaint Louverture, Paris, Présence Africaine, 1981, p. 243. 
du général Richepanse pour la Guadeloupe - prévoyaient la déportation en France des principaux chefs insulaires, le désarmement de l'armée noire et un prompt rétablissement de l'esclavage.

A Saint-Domingue, il fallait d'abord se débarrasser de l'homme fort du pays - ce qui fut fait par traîtrise. Toussaint tomba, en effet, dans un guet-apens le 7 juin 1802, et fut embarqué aussitôt sur un navire à destination de la métropole ; il fut emprisonné dans le Jura au Fort de Joux où il mourut en avril 1803. Mais dans le prolongement de l'élimination de Toussaint-Louverture, se produisit dans l'île un soulèvement général regroupant «gens de couleur» et esclaves émancipés qui n'étaient pas disposés à retrouver leur ancienne condition servile. La nouvelle de l'échec de l'insurrection de Delgrès en Guadeloupe et l'annonce officielle du rétablissement de l'esclavage accéléra considérablement à Saint-Domingue l'ampleur du mouvement insurrectionnel qui devint une véritable guerre, laquelle allait durer deux ans. Les généraux de l'armée noire, Jean-Jacques Dessalines et Henri Christophe, prirent la relève de Toussaint Louverture. Sous leur direction et notamment celle de Dessalines, leur général en chef depuis mai 1803, cette armée réussit à défaire l'armée française qui restait pourtant invaincue en Europe. Les dernières troupes françaises, décimées par les attaques d'une armée luttant pour «la liberté ou la mort» selon le cri de ralliement des soldats, submergées par le nombre, et qui plus est prises à revers par la flotte anglaise, durent capituler à la fin de novembre 1803. La colonie française de SaintDomingue avait vécu. Le $1^{\text {er }}$ janvier 1804, Dessalines proclama l'indépendance d'Haïti la noire. C'était la première fois dans l'Histoire qu'une révolte d'esclaves - sans équivalent depuis celle de Spartacus était victorieuse et gagnait une guerre contre une grande puissance qui dominait alors la quasi-totalité de l'Europe.

L'onde de choc de cet événement sans précédent allait se faire sentir dans toute la Caraïbe et accélérer le processus de destruction du système esclavagiste dans les îles voisines qui allaient être en proie à des soulèvements toujours plus nombreux Elle eut un écho jusqu'en Amérique latine dont les dirigeants de lutte de libération comme Miranda, ou Bolivar trouvèrent asile et aide en Haïti. 
Le spectre d'Haïti pétrifia l'oligarchie antillaise au point de la persuader de modérer ses traditionnelles velléités autonomistes, sinon séparatistes. Bon gré, mal gré, cette oligarchie remit désormais son destin entre les mains de l'Etat français, quel qu'il fût, pour pérenniser ses intérêts dans une conjoncture marquée par une montée générale de l'abolitionnisme.

C'est dans ce contexte troublé qu'intervint une décision de l'Angleterre qui devait porter un premier coup fatal au système esclavagiste. Le 25 mars, Londres mettait fin à la traite. Le Portugal et les Etats-Unis firent de même aussitôt après. Cette décision anglaise mettait évidemment mal à l'aise le pays des «Droits de l'Homme », qui la maintenait, et rendait à terme, sa position intenable. Sur le moment, Napoléon fit mine de dédaigner la portée de cette mesure. Il faut dire qu'il venait avec difficultés de rétablir l'esclavage dans les îles. Mais, ce ne fut pas pour longtemps car le domaine colonial français d'Amérique allait s'effondrer sous les coups de l'ennemi espagnol mais surtout anglais. La Martinique et la Guadeloupe, en ce qui les concerne, furent pour la seconde fois occupées par les Anglais à partir de 1809 et ce jusqu'à la Restauration, en 1815.

La chute de Napoléon ne changea pas précisément le statut des Antilles. Les colons, étant donné leur rôle durant la Révolution, leurs relations étroites avec la Noblesse d'Ancien Régime, obtinrent sans difficulté que l'article 73 de la charte constitutionnelle du 4 juin 1814 mît les colonies hors du droit commun, comme si rien ne s'était passé depuis 1789 .

Mais contrairement à la situation qui prévalait durant la période révolutionnaire, la France désormais se trouvait bien en retard, en ce qui concernait le problème de l'esclavage, par rapport aux autres puissances européennes : la Grande-Bretagne et le Danemark avaient aboli dès 1807 la traite négrière, la Suède en 1813, les Pays-Bas en 1814. Lors du Congrès de Vienne (1814-1815), sous la pression de l'Angleterre, les puissances européennes adoptèrent une déclaration commune dans laquelle elles acceptaient toutes d'abolir la traite. Louis XVIII fut contraint de l'avaliser. Ce fut le premier coup sérieux porté au système esclavagiste dont l'une des bases était évidemment la traite 
négrière. Désormais, l'esclavage ne pouvait se maintenir, si l'on peut dire, que par la seule croissance démographique des esclaves des colonies.

Un des premiers actes du Napoléon des «Cent Jours» fut d'entériner ce nouvel état de fait, sans doute autant pour affermir sa position. Il revenait ainsi sur la décision du Premier Consul qu'il avait été en 1802 lorsqu'il était «dans des vues qui, sans doute, n'avaient pas pour objet le seul bien de l'humanité ${ }^{4}$. Cinq articles d'un décret tranchant abolissaient désormais la traite. Chez les armateurs cet engagement avait évidemment provoqué la consternation. Louis XVIII à peine revenu après l'exil de Napoléon à l'île de Saint-Hélène, ils réclamèrent «justice», suppliant pour une «branche de commerce dont le besoin est si bien ressenti dans les colonies ». Pour l'Angleterre cependant, la décision de Napoléon était définitive. Sa remise en cause ne pouvait être acceptée tout au moins sur le plan des principes. Aussi, le 30 juillet 1815, Louis XVIII confirma-t-il l'engagement de l'abolition de la traite ; ce qui n'empêcha pas la poursuite d'une traite clandestine qui perdura jusque dans les années 1830. L'Angleterre eut beau faire pression pour imposer le «droit de visite » de tout navire suspecté afin de vérifier s'il ne transportait pas des esclaves, cela n'aboutit jamais. Les autorités françaises, complaisantes, préférèrent fermer les yeux sur ce trafic en théorie illicite.

Les choses étaient malgré tout en train de changer en profondeur dans les colonies et les événements qui se déroulèrent aux Antilles dans les années 1820, sous la Restauration, ne furent sans doute pas étrangers au fait que la question de l'assimilation intéressa de nouveau la métropole. Cela se fit via la réouverture du débat sur l'égalité civique, lequel anticipait celui qui aurait lieu sur l'abolition définitive de l'esclavage dont l'Abbé Grégoire s'était depuis toujours fait le porte-parole. Mais la reconstitution du mouvement anti-esclavagiste en France durant la Restauration fut une tâche difficile, en partie parce que Grégoire était alors stigmatisé comme régicide puisqu'il avait voté

${ }^{4}$. Cf. Serge Daget, «l'abolition de la traite des noirs en France de 1814 à $1831 »$, in Cahiers d'Etudes Africaines, Vol. 11, n 41, p. 22. 
la mort de Louis XVI, ce qui portait préjudice au mouvement abolitionniste tout entier.

\section{LA BATAILLE POUR LA LIBERTE ET L'EGALITE CIVIQUE COMME LUTTE POUR LA CITOYENNETE FRANÇAISE SOUSLA RESTAURATION ET LA MONARCHIE DE JUILLET}

Par delà les régressions induites par l'Empire et la Restauration, le bouleversement révolutionnaire de la société française avait marqué, aux Antilles, le début d'une période de lutte politique décisive via la revendication de l'assimilation. La première moitié du XIX ${ }^{\mathrm{e}}$ siècle vit s'aiguiser la lutte des «libres de couleur» pour l'égalité civique. De manière générale, ceux-ci commençaient à former une classe nombreuse, instruite et plus consciente que jamais de ses droits bafoués. Les affrontements allaient donc se multiplier dans la société esclavagiste agonisante.

Les « libres de couleur» étaient naturellement attirés par ce qui se passait dans l'île de Saint-Domingue, devenue Haïti. Mais les «libres » ne furent pas seuls à combattre le système colonial ségrégationniste. Peu à peu, les esclaves entrèrent dans la lutte pour la liberté, de plus en plus en union avec les «libres », qui étaient en général confondus dans les textes avec les esclaves puisqu' officiellement et surtout juridiquement la distinction n'existait pas. Dans cette première partie du $\mathrm{XIX}^{\mathrm{e}}$ siècle et juste après la Restauration, une succession de soulèvements se produisirent notamment à la Martinique, qui furent sans aucun doute pour beaucoup dans l'abolition de l'esclavage en 1848.

En octobre 1822, éclata à la Martinique la révolte d'esclaves du Carbet, qui fit deux morts et sept blessés dont deux «hommes de couleur libres » et qui déboucha sur une vingtaine de condamnations à mort à la clé. En décembre 1823 surtout, survint «l'affaire Bissette». Une brochure de trente-deux pages, imprimée en France et intitulée $D e$ la situation des gens de couleur aux Antilles françaises, fut introduite clandestinement à la Martinique. Il s'agissait d'une pétition collective des «Gens de couleur libres » martiniquais en faveur de l'égalité des 
droits. Cette brochure allait, aux Antilles, faire l'effet d'un coup de tonnerre. La paternité du texte fut entre autres attribuée à un notable de couleur, Cyrille-Auguste Bissette qui fut accusé d'avoir fait circuler l'opuscule en vue de fomenter une insurrection contre les planteurs blancs. A la Martinique, ces derniers crièrent au scandale et dénoncèrent son introduction aux autorités de l'île comme une conspiration. Ils firent savoir au Gouverneur qu'ils considéraient l'écrit incriminé comme «séditieux », que le ou les coupables supposés devaient immédiatement être poursuivis et sévèrement punis. Il n'y avait rien dans la brochure que tout le monde ne connût déjà. La réalité était que les planteurs blancs ne pouvaient se résoudre à accepter de se voir un jour les égaux des «gens de couleur», et ce, même s'ils étaient déjà des libres. Sous la pression des milieux blancs, le pouvoir colonial « découvrit» fort opportunément un «complot». A l'issue de plusieurs arrestations, la Cour Royale de la Martinique condamna Cyrille Bissette ainsi que deux autres compagnons d'infortune dénommés Louis Fabien et Jean-Baptiste Volny, aux galères à perpétuité. Au terme d'une intense campagne de solidarité en France avec les condamnés qui avaient été victimes d'un jugement inique, la Cour de Cassation finit par revenir sur la première sentence. Seul Bissette, «convaincu d'avoir semé une brochure séditieuse et diffamatoire », fut finalement frappé d'une peine de bannissement de 10 ans. Cette affaire constitua un tournant par l'importance de l'écho qu'elle eut dans l'opinion publique métropolitaine. Les événements qui suivirent durant la Monarchie de Juillet ne firent que souligner, aux yeux du pouvoir français, l'urgence des réformes à entreprendre pour éviter un embrasement général de la situation. En février 1831, en effet, un soulèvement d'esclaves à Saint-Pierre conduisit le Gouverneur à mettre la colonie en état de siège. En décembre 1833, enfin, une nouvelle révolte d'esclaves éclata à Grande-Anse (Martinique) : à l'issue d'un procès expéditif, sur les 82 personnes retenues comme accusées, 15 des révoltés furent condamnés à mort, 22 aux travaux forcés et 25 à la déportation. Dans un contexte où les autres puissances coloniales de la région avaient déjà aboli l'esclavage dans leurs colonies, il était devenu impossible pour la France de s'en tenir au statu quo en la matière. Mais la Monarchie de Juillet, qui souhaitait une émancipation seulement progressive, probablement en 
raison du problème que constituerait un éventuel « dédommagement », avait toujours refusé de s'attaquer de front à la question de l'esclavage. Ce problème ne devait être réglé, sous la pression de dramatiques événements, qu'à l'issue de la Révolution de 1848.

\section{L'ABOLITION DE L'ESCLAVAGE PAR LA II ${ }^{\mathrm{E}}$ REPUBLIQUE ISSUE DE LA REVOLUTION DE 1848}

Il appartint au gouvernement provisoire issu de la révolution de 1848, d'instituer une commission pour préparer l'acte d'émancipation immédiate dans toutes les colonies de la République. Victor Schœlcher, depuis longtemps partisan de l'abolition immédiate - dans le cadre du «Comité pour la libération des Noirs » créée en 1832, puis de la «Société pour l'abolition de l'esclavage » fondée en 1834 -, président en titre de cette commission, et nommé par François Arago Sous-secrétaire d'Etat aux Colonies, écrit à l'époque dans le rapport adressé au ministre de la Marine et des Colonies, qu'en donnant la France comme patrie, et tous les droits de citoyens comme héritage, à ceux qui hier encore étaient asservis à d'autres, la France témoigne assez hautement qu'elle n'exclut personne de son immortelle devise : «Liberté, Egalité, Fraternité ».

Le 4 mars 1848, le décret préparatoire de l'abolition entérinait le principe de la suppression de l'esclavage dans les colonies françaises. Le décret d'abolition était finalement voté par le Gouvernement provisoire le 27 avril 1848. Le texte du décret était le suivant :

«Le Gouvernement Provisoire de la République : considérant que l'esclavage est un attentat à la dignité humaine,

«Qu'en détruisant le libre-arbitre de l'homme il supprime le principe naturel du droit et du devoir,

«Qu'il est une violation flagrante du dogme républicain « Liberté, Egalité, Fraternité », 
déclare dans son article premier :

«l'esclavage sera entièrement aboli dans toutes les colonies et possessions françaises, deux mois après la promulgation du présent décret dans chacune d'elles. A partir de la promulgation du présent décret dans les colonies, tout châtiment corporel, toute vente de personnes non libres seront absolument interdits ».

Et dans son article 5 :

«L'Assemblée Nationale réglera la quotité de l'indemnité qui devra être accordée aux colons ».

Une indemnité coloniale, accordée par le gouvernement aux anciens propriétaires d'esclaves par une loi du 30 avril 1849, sera versée partie en numéraire, partie sous la forme du capital de base des banques coloniales ouvertes en Guadeloupe et en Martinique en 1853 : La Société de Crédit Colonial en 1861 qui devint le Crédit Foncier Colonial en 1863 (futur Crédit Foncier de France).

Schœlcher, animé par ses convictions socialistes, a agi avec le réalisme qu'imposaient certaines pressions conservatrices, voire réactionnaires - ce que prouve l'article 5 -, mais sans jamais perdre de vue le but qu'il s'était fixé pour aider à la libération des opprimés dont il s'était fait depuis longtemps le défenseur. Dans un discours prononcé le 21 juillet 1945, jour de la Saint-Victor, traditionnellement consacré à la mémoire de Schœlcher, Césaire s'exprima en ces termes : «...la belle œuvre de Schœlcher. Une œuvre non écrite et pourtant vivante. Une œuvre publiée par des milliers de visages et imprimée dans des milliers de cœurs : le 27 avril 1848, un peuple qui depuis des siècles piétinait sur les degrés de l'ombre, un peuple que depuis des siècles le fouet maintenait dans les fosses de l'histoire, un peuple torturé depuis des siècles, un peuple humilié depuis des siècles, un peuple à qui on avait volé son pays, ses dieux, sa culture, un peuple à qui les bourreaux tentaient de ravir jusqu'à son nom d'homme, ce peuple-là, le 27 avril 1848, par la grâce de Victor Schœlcher et la volonté du peuple français, rompait ses chaînes et au prometteur soleil d'un printemps 
inouï, faisait irruption sur la grand-scène du monde ${ }^{5} \gg$. Pour Césaire, avril 1848, c'est en quelque sorte l'émergence d'un peuple désormais sujet de son histoire.

En effet, les opprimés, émancipés par le vote du 27 avril 1848, anticipèrent l'application du décret ; ce sont les esclaves martiniquais qui devaient conquérir par eux-mêmes la liberté avant même d'attendre l'arrivée de ce décret - il fallait alors près de trente jours de voyage transatlantique -, en déclenchant la grande insurrection du 22 mai 1848.

La nouvelle de l'existence du décret préparatoire du 4 mars n'arriva dans les îles qu'au début du mois d'avril. Tous les témoins sur place en Guadeloupe et à la Martinique, soulignèrent l'impatience et les espérances suscitées par l'avènement de la République qui avait rendu l'atmosphère quasi-électrique, ainsi que par l'attente de l'émancipation. De multiples incidents survinrent dans les deux colonies au cours du mois d'avril où les esprits s'échauffèrent. La tension monta surtout dans les villes où des manifestations populaires eurent lieu dans la seconde quinzaine d'avril. Or, les navires de France n'apportaient toujours pas le décret d'émancipation tant attendu, et pour cause, puisqu'il ne fut voté à Paris que le 27 avril. Il suffisait d'une étincelle pour mettre le feu aux poudres.

En mai 1848, les esclaves s'insurgèrent en Guadeloupe et à la Martinique. A la Martinique, des troubles particulièrement graves se produisirent le 22 au Prêcheur, à Saint-Pierre, qui firent 25 tués et une cinquantaine de blessés. Dans le prolongement de cet affrontement, des émeutes éclatèrent dans plusieurs ateliers du sud (au Robert, au François); l'insurrection se propagea ensuite à Grand'Anse (Lorrain), Trinité, Gros-Morne, Marin, Lamentin, Sainte-Marie et surtout à Fortde-France le 23 mai, alors que le Gouverneur Rostolan, invité par le Conseil municipal de Saint-Pierre, était absent depuis le 22. Ces troubles ayant provoqué le mort de plusieurs dizaines de personnes amenèrent le Gouverneur Rostolan tout juste débarqué le 22 à anticiper l'arrivée du décret parisien et à prendre dès le 23 mai l'arrêté suivant :

5. Cf. Marie-José Jolivet, «La construction d'une mémoire historique à la Martinique », in Cahiers d'Etudes Africaines, 107 - 108, XXVII, n 3 - 4, p. 300. 
«Considérant que l'esclavage est aboli en droit et qu'il importe à la sécurité du pays de mettre immédiatement à exécution la décision du gouvernement de la métropole pour l'émancipation générale dans les colonies françaises, arrête :

Art. $1^{\text {er }}$ :

«L'esclavage est aboli à partir de ce jour à la Martinique ».

Art. 2 :

«Le maintien de l'ordre est confié au bon esprit des anciens citoyens français. Ils sont en conséquence invités à prêter main-forte à tous les agents de la force publique pour assurer l'exécution des lois ».

La lutte des esclaves martiniquais fut donc directement à l'origine de cette décision. Ce qu'est contraint de reconnaître d'ailleurs le Gouverneur Rostolan lui-même.

L'insurrection martiniquaise accéléra, en outre, l'abolition de l'esclavage en Guadeloupe où le Gouverneur de l'île, ayant appris les événements de la Martinique, et craignant des troubles comparables, décréta également l'abolition immédiate et sans délai.

En Guadeloupe, en effet, le gouverneur, après avoir convoqué le Conseil privé le 27 mai à 6 heures du matin et entendu une députation du Conseil municipal de Pointe-à-Pitre, prit un arrêté similaire :

« Nous, Gouverneur de la Guadeloupe, considérant que, par le bon esprit dont elle a fait preuve, la population esclave s'est montrée digne du bienfait de la liberté, (...) arrêtons :

Art. $1^{\text {er }}$ :

«L'esclavage est aboli».

Art. 2 :

«L'indemnité, due légitimement aux propriétaires, est placée sous la sauvegarde de l'honneur français et recommandée à la justice de l'assemblée Nationale ». 
Art. 3 :

«Le commandant militaire et les chefs d'administration sont chargés de l'exécution du présent arrêté ».

L'Assemblée Constituante n'apprit que le 22 juin ces faits et ces décisions par l'entremise du Ministre de la Marine et des Colonies. En réalité c'est seulement à la Guyane que tout se passa sans trop d'agitation. Le décret d'abolition du 27 avril y arriva le 10 juin. L'esclavage y fut officiellement aboli le 10 août 1848.

C'est donc sous l'irrésistible pression insurrectionnelle dans les îles que furent pris ces décrets lesquels anticipaient dans l'urgence l'arrivée du décret voté à Paris le 27 avril 1848. L'insurrection martiniquaise de mai fut, dans tous les sens du terme, une Révolution martiniquaise et pour ainsi dire l'acte de naissance d'un «peuple martiniquais », acteur de sa propre histoire. Il sera un élément déterminant de la construction d'une mémoire nationale martiniquaise. C'est ce qui ressort d'un discours prononcé par Césaire, en tant que maire de Fort-de-France, le 22 mai 1971, à l'occasion de l'inauguration d'une statue de la liberté et d'une place du 22 mai, discours dont une phrase éclaire le sens de l'entreprise : «...Voilà l'événement que nous célébrons aujourd'hui (...) : une liberté non pas octroyée mais arrachée de haute lutte ; une émancipation non pas concédée mais conquise, et qui enseigne à tous, et d'abord aux Martiniquais eux-mêmes, que s'il est vrai que la Martinique est une poussière, il y a cependant des poussières habitées par des hommes qui méritent le nom d'hommes. Et cette assurance, voyez-vous, est de celles qui nous autorisent à regarder le présent avec plus de fermeté et à toiser l'avenir avec plus d'insolence $»^{6}$.

De 1848 au coup d'Etat du 2 décembre 1851, s'instaura aux Antilles une vie démocratique, fondée en droit sur le suffrage universel et une certaine assimilation institutionnelle entre les colonies et la métropole. Le Gouverneur Rostolan fut remplacé début juin par un Commissaire général de la République martiniquais, proche de

6. Cf. Marie-José Jolivet, «La construction d'une mémoire historique à la Martinique », in Cahiers d'Etudes Africaines, 107 - 108, XXVII, n 3 - 4, p. 300. 
Schœlcher, François-Auguste Perrinon. Nommé au poste de chef administratif et militaire de son île natale - siège du gouvernement général des Antilles françaises -, il fut le premier antillais de couleur promu à un poste de cette nature. Perrinon n'avait pas seulement à appliquer le décret d'abolition, mais aussi les autres dispositions du 27 avril.

Par-delà l'abolition de l'esclavage, en effet, ces décrets proclamaient l'assimilation des vieilles colonies à la métropole. Le décret d'émancipation accordait une liberté «immédiate» aux esclaves, contrairement à l'abolition «progressive» britannique et faisait des «nouveaux libres » de « nouveaux citoyens » (hommes âgés de plus de 25 ans) qui devaient élire au suffrage universel leurs représentants à l'Assemblée Nationale. Pratiquant une politique résolument assimilationniste, la Seconde République supprimait les conseils coloniaux et décidait que les colonies enverraient des représentants à l'Assemblée Nationale. En septembre enfin, les conseils municipaux, qui avaient été créés le 12 juin 1837, furent réorganisés sur la base du suffrage universel.

Sur le fond de cette histoire, se détache, omniprésente, une figure politique emblématique, celle de Schœlcher qui est devenu pour la postérité le symbole de cette évolution progressiste. Sa popularité aux Antilles avait pu se mesurer aux élections de 1848, où Martiniquais et Guadeloupéens à l'unisson voulurent en faire l'un des trois députés que chaque île devait envoyer à l'Assemblée Constituante. Dans une adresse à ses électeurs de la Guadeloupe et de la Martinique, en date du 29 septembre 1848, il aura ces mots : «purifiées de la servitude, les colonies sont désormais une partie intégrante de la Métropole; disons mieux, il n'y a plus de colonies, il n'y a que des Départements d'Outre-Mer régis par les mêmes lois que ceux du continent ». Il opta finalement pour la Martinique. Mais l'année suivante aux élections législatives de 1849, les Martiniquais lui préférèrent un homme du pays, Bissette, resté célèbre pour avoir lutté, dès la Restauration, en faveur de l'égalité des droits pour les « hommes de couleur libres » dont il faisait partie, et pour avoir rallié ensuite les thèses abolitionnistes. A l'approche des élections de 1849 qui devaient envoyer désormais deux députés par colonies à la Nouvelle Assemblée 
Législative, Bissette fit une alliance tactique avec les planteurs blancs - ce qui le fit par la suite désigner comme traître à la cause - et supplanta Schœlcher, lequel fut néanmoins réélu, en même temps que Perrinon, par les Guadeloupéens.

L'abolition de l'esclavage, même si elle avait abouti à la proclamation immédiate d'une égalité formelle absolue dans le domaine civil, ne résolvait pas pour autant la question de l'application concrète des droits politiques. A partir de novembre 1848 et jusqu'à décembre 1851, il y eut un sabotage de l'œuvre progressiste du Gouvernement provisoire. Les grands propriétaires se ressaisirent en même temps que la réaction antirépublicaine se dessinait de plus en plus nettement en France. Les trois années qui suivirent l'abolition de l'esclavage virent s'effriter l'œuvre de la Révolution de 1848 et l'œuvre progressiste de Schœlcher. Les principales dispositions du décret du 27 avril 1848 furent malaisément appliquées. Tous les volets de la République sociale étaient bafoués. Le droit à la sécurité sociale ne fut jamais exécuté. La liberté de la presse était laissée à la discrétion des gouverneurs. Seuls les journaux qui pouvaient payer un cautionnement, donc ceux qui représentaient les intérêts conservateurs, avaient pu subsister. Cette libéralisation de la presse fut donc une libéralisation avortée. Quant au droit à l'instruction, il était conditionné par l'obligation, pour les affranchis, de rester attachés au sol des grandes habitations. Il ne restait de tout l'édifice schœlchérien que l'abolition de l'esclavage proprement dite. Quant aux libertés publiques indistinctement reconnues aux habitants de la Guadeloupe et à la Martinique, elles allaient être rapidement supprimées par le Second Empire.

De fait, il fallut encore attendre, par delà la régression que constitua le Second Empire en matière de droits civils et politiques, l'avènement de la $\mathrm{III}^{\mathrm{e}}$ République pour voir resurgir les revendications d'assimilation. En arrière-plan de telles revendications se profilait la départementalisation qui apparaissait de plus en plus comme inéluctable à terme. Le Conseil général de la Martinique en avait d'ailleurs très vite fait son cheval de bataille. Dès 1874, en effet, les élus du Conseil général de la Martinique émirent à l'unanimité le vœu de faire reconnaître les colonies, «comme terres françaises, parties 
intégrantes de la République ». Cette demande avait été constamment renouvelée : en 1882, 1885, 1889. Le Conseil général intervint de nouveau en 1925, pour une assimilation «mitigée », et enfin pour une assimilation «intégrale » en 1938. Il s'agissait là des deux formules d'assimilation («mitigée » et «intégrale»), parmi les solutions proposées par le rapport Leconte, établi en 1925 à la demande du ministre des Colonies, après consultation des gouverneurs et des assemblées de Martinique, Guadeloupe et Guyane, pour résoudre la question sans cesse reposée du statut. Faut-il rappeler qu'en 1935, Joseph Lagrosillière, Président du Conseil Général de la Martinique, n'avait pas hésité à s'écrier : «Vivent désormais les Antilles, la Guyane et la Réunion en départements français », tout juste avant de déposer une proposition de loi en ce sens. Cette demande récurrente d'assimilation aboutira finalement, après le sombre intermède vichiste qui avait rétabli la discrimination raciale, en 1945. Personne au lendemain de la Seconde Guerre Mondiale, ne douta que le moment ne fût venu de réaliser cette assimilation pour laquelle s'étaient battues plusieurs générations de Martiniquais. Elle se concrétisa, immédiatement après 1945, avec la départementalisation des «Quatre Vieilles ».

\section{CONCLUSION}

C'est d'une certaine manière le même processus assimilationniste que celui qui a débouché sur l'abolition de l'esclavage en 1848 qui trouva son ultime prolongement dans la transformation, par la loi du 19 mars 1946, des «quatre vieilles » colonies qu'avaient été jusque-là la Martinique, la Guadeloupe, la Guyane ainsi que la Réunion, en départements français au même titre que les départements métropolitains. 


\section{BIBLIOGRAPHIE}

\section{Ouvrages :}

ALPHONSE R., Le conflit des tendances «assimilationnistes et antiassimilationnistes » à la Guadeloupe et à la Martinique, Paris, Publications de la Fondation Nationale de Sciences Politiques, 1966.

BAngou Henri, La Révolution et l'esclavage à la Guadeloupe (1789 - 1802), Paris, Messidor - Editions Sociales, 1989.

BenOt Yves, La Révolution française et la fin des colonies, Paris, La Découverte, 1988.

BlEt Henri, France Outre-Mer, l'œuvre coloniale de la Troisième République, Paris, Editions Arthaud, 1950.

Cesaire Aimé, Discours sur le colonialisme, Paris, Editions Réclame, 1950.

Constant Fred, Daniel Justin Eds., 1946-1996, Cinquante ans de départementalisation Outre-mer, Paris, L'Harmattan, 1997.

Darmiant, De la situation des gens de couleur libre aux Antilles, Paris, J. McCarthy, 1823.

DARSIERES Camille, Des origines de la nation martiniquaise, Fort-de-France, Désormeaux, 1974.

Deville Robert, GeORges Nicolas Eds., Les départements d'outre-mer : l'autre décolonisation, Paris, Découvertes Gallimard, 1996.

GuERIN Daniel, Les Antilles décolonisées, Paris, Présence Africaine, 1956.

JAMES C.-R., Les Jacobins noirs, Toussaint Louverture et la révolution de Saint-Domingue, Paris, Gallimard, 1949.

LeIRIS Michel, Contacts de civilisations en Martinique et en Guadeloupe, Paris, UNESCO, Gallimard, 1955.

LEPINE Edouard (de), Questions sur l'histoire antillaise : trois essais sur l'Abolition, l'Assimilation, l'Autonomie, Fort-de-France, Désormeaux, 1978.

Nicolas Armand, Histoire de la Martinique, 2 Vol., Paris, L'Harmattan, 1996.

Pluchon Pierre Ed., Histoire des Antilles et de la Guyane, Paris, Privat, 1982.

SABLE Victor, La transformation des Isles d'Amérique en départements français, $_{2}$ Paris, Editions Larose, 1955.

SCHELCHER Victor, Abolition de l'esclavage : examen critique du préjugé contre la couleur des Africains et sang-mêlés, Paris, Pagnerre, 1840. 


\section{Articles :}

BLERALD Alain Philippe, «La citoyenneté française aux Antilles et ses paradoxes », in 1946-1996, Cinquante ans de départementalisation Outre-mer, Constant Fred, Daniel Justin Eds., Paris, L'Harmattan, 1997, pp. 193 - 204.

BRETTE A., «Les gens de couleur libres et leurs députés en 1789 », $\mathrm{La}$ Révolution française, Vol. 29, pp. 326 - 345 et 385 - 407, 1895.

CotTias Myriam, "'L'oubli du passé" contre la "citoyenneté" : troc et ressentiment à la Martinique (1848-1946)», in 1946-1996, Cinquante ans de départementalisation Outre-mer, Constant Fred, DANIEL Justin Eds., Paris, L'Harmattan, 1997, pp. 293 - 313.

Colin Claude, LANFrey Jean-François, «La Martinique, département ou colonie ? », Les Temps Modernes, $\mathrm{n}^{\circ}$ 345, avril 1975, pp. 1339 - 1389.

DAGET Serge, «L'abolition de la traite des noirs en France de 1848 à 1831 », Cahiers d'Etudes Africaines, Vol. 11, n 41, 1971, pp. 14 - 58.

FENET A., «Assimilationnisme politique et réalité juridique dans la politique coloniale française », Pluriel, $\mathrm{n}^{\circ}$ 17, 1979, pp. 47 - 55

FREDJ Jacques, «L'assimilation dans l'histoire antillaise», Les Temps Modernes, $\mathrm{n}^{\circ} 441$ - 442, avril - mai 1983, pp. 1836 - 1852.

GiRAult A., «Le problème colonial : assujettissement, autonomie, ou assimilation », in Revue du Droit Public et de la Science Politique, mai - juin 1894, p. 52.

JoLIVET Marie-José, «La construction d'une mémoire historique à la Martinique : du Schœlchérisme au marronisme », Cahiers d'Etudes Africaines, Vol. 27, $\mathrm{n}^{\circ} 3$ - 4, 1987, pp. 287 - 309.

MANChUELLE François, «Le rôle des Antillais dans l'apparition du nationalisme culturel en Afrique noire francophone », Cahiers d'Etudes Africaines, Vol. 32, n 3, 1992, pp. 375 - 408.

MonNerot J., «L'assimilation aux départements de la métropole », Revue de la Martinique, $\mathrm{n}^{\circ}$ 14, septembre 1930, pp. 8 - 31.

SAINT-CYR Philippe, «Du pacte colonial au pacte départemental», Les Cahiers du CERAG, s. d., n ${ }^{\circ} 29$, pp. 1 - 27.

SEMIDEI Manuela, "De l'Empire à la décolonisation», Revue Française de Science Politique, Vol. 16, $\mathrm{n}^{\circ}$ 1, 1966.

WiLLIAM Jean-Claude, «Les origines de la loi de départementalisation », Historial Antillais, Vol. 6, Editions Dejani, 1980, pp. 50 - 61. 


\section{Résumé}

A partir de la Révolution française, l'assimilation devait se présenter comme la réponse des « Gens de couleur» à l'autonomisme ségrégationniste et oppressif des colons. Ils ne faisaient là que pousser dans ses ultimes conséquences l'idéologie universaliste de la «Déclaration des droits de l'homme et du citoyen »d'août 1789 , même si la Révolution, curieusement conservatrice par certains aspects, tarda à concrétiser les espoirs qu'elle avait pu susciter. Par delà les régressions induites par l'Empire et la Restauration, les bouleversements de la période révolutionnaire en Métropole avaient marqué, aux Antilles, le début d'une phase de lutte politique décisive qui devait déboucher sur l'abolition définitive de l'esclavage en 1848. Et c'est, d'une certaine manière, le même processus assimilationniste qui trouvera son ultime prolongement dans la transformation, par la loi du 19 mars 1946, des «quatre vieilles » colonies qu'avaient été jusque-là la Martinique, la Guadeloupe, la Guyane ainsi que la Réunion, en départements français au même titre que les départements métropolitains.

\section{Abstract}

Since French Revolution, Assimilation was thought as the correct response from "Colored People» to the colonial oppressive and segregative policy of selfgoverning vis-à-vis the Metropolis. "Colored People» were thus stretching the logics of the Déclaration des droits de l'homme et du citoyen of August 1789 to the limits of its outcome, even though French Revolution astonishingly conservative as it were, did not fulfill immediatly the hopes it was supposed to bring along. Although the Empire and Restauration policy led to the old slavery times, disruptions enticed during the Revolution in France, gave rise to a decisive political struggle wich would endly lead, in Antilles, to the abolition of slavery in 1848. It is in a way, the same process at work wich led up to the transformation through the Law of March 1946 to the «Four Oldies» - Martinique, Guadeloupe, French Guiana and Reunion island - into French départements in the same way as départements of Metropole.

\footnotetext{
Mots-clés $-\cdots-\cdots-\cdots-\cdots-\cdots, \cdots-\cdots, \quad-\cdots, \cdots-\cdots, \cdots-\cdots, \cdots-\cdots$ Keys-words

Antilles - Assimilation Autonomisme - Départementalisation Emancipation - Esclavage - Guadeloupe Martinique - République Révolution française - Saint-Domingue
Antilles - Assimilation - Autonomism - Départementalisation - Emancipation - French Revolution - Guadeloupe - Slavery - Martinique - Republic - Saint Domingue - 